\title{
LHCf plan for proton-oxygen collisions at LHC
}

\section{E. Berti ${ }^{a, b, *}$ and L. Bonechi ${ }^{b}$ on behalf of the LHCf Collaboration}

(a complete list of authors can be found at the end of the proceedings)

${ }^{a}$ University of Firenze, Via G. Sansone 1, Sesto Fiorentino, Firenze, Italy

${ }^{b}$ INFN Section of Florence, Via B. Rossi 2, Sesto Fiorentino, Firenze, Italy

E-mail: eugenio.berti@fi.infn.it

The LHCf experiment is designed to provide precise measurements of very forward neutral particle production from high energy proton-proton, proton-ion and ion-ion collisions. This information is necessary to test and tune hadronic interaction models used by ground-based cosmic rays experiments to extract the average composition of Ultra High Energy Cosmic Rays. In order to reach this goal, LHCf makes use of two small sampling calorimeters installed in the LHC tunnel at $\pm 140 \mathrm{~m}$ from Interaction Point 1 , both able to detect neutral particles having pseudo-rapidity $\eta>$ 8.4. In LHC Run I and II, the LHCf experiment acquired data relative to p-p collisions at $\sqrt{s}=0.9,2.76,7$ and $13 \mathrm{TeV}$, and $\mathrm{p}-\mathrm{Pb}$ collisions at $\sqrt{s_{N N}}=5.02$ and $8.16 \mathrm{TeV}$. Forward production from $\mathrm{p}-\mathrm{p}$ and $\mathrm{p}-\mathrm{Pb}$ collisions are not directly applicable to the tuning of the model used to simulate extensive air showers, since the first interaction between a cosmic ray and an atmospheric nucleus generally involves a light nucleus, like N or O. In LHC Run III, we will have the unique opportunity to directly measure forward production from high energy p-O collisions, without the need to obtain this information by interpolating the measurements from $\mathrm{p}-\mathrm{p}$ and $\mathrm{p}-\mathrm{Pb}$ collisions. In this contribution, we discuss the importance of such a measurement, focusing on all the benefits in terms of a more direct and complete input for model tuning, and on the operation plans, including the importance to take data both from high energy p-O and O-O collisions.

$37^{\text {th }}$ International Cosmic Ray Conference (ICRC 2021)

July 12th - 23rd, 2021

Online - Berlin, Germany

\footnotetext{
*Presenter
} 


\section{Introduction}

In recent years, several results obtained by large ground-based cosmic ray experiments, like Pierre Auger Observatory [1] and Telescope Array [2], gathered the attention of the scientific community. In particular, the average composition of Ultra High Energy Cosmic Rays (UHECR), cosmic rays above $10^{18} \mathrm{eV}$, resulted to be consistent with an admixture of light nuclei, but not with protons only. However, even if this result is qualitatively clear, the quantitative estimation of the average composition suffers of large uncertainties. These uncertainties are not due to the experimental technique, but to the hadronic interaction model used to simulate the Extensive Air Showers (EAS), which are formed by the interaction of an UHECR with the atmosphere. In order to reduce this theoretical uncertainty, it is necessary to take high energy calibration data that can be used to tune these models. The Large Hadron Collider (LHC [3]) at CERN is the most suitable place where we can perform these measurements, since a p-p collision at the center of mass energy of $\sqrt{s}=13 \mathrm{TeV}$ allows us to study a configuration that is equivalent to the first interaction of $9 \times 10^{16} \mathrm{eV}$ proton cosmic ray with an atmospheric proton at rest. All the key parameters used to model EAS development can be measured at the LHC: inelastic cross section, multiplicity of secondaries, and forward energy distributions, from which one can derive the average inelasticity. The first two quantities are easily accessible to the four central detectors and roman pot detectors, but forward production, which is so important for EAS development since the energy flow is peaked in this region, can be measured only by a dedicated forward experiment. The main aim of the LHC forward (LHCf [4]) experiment is to give important information for the calibration of hadronic interaction models by accurate measurements of very forward particle production in $\mathrm{p}$ - $\mathrm{p}, \mathrm{p}$-ion and ion-ion collisions. In LHC Run I and II, LHCf acquired data relative to p-p collisions at $\sqrt{s}=0.9$, 2.76, 7 and $13 \mathrm{TeV}$, and $\mathrm{p}-\mathrm{Pb}$ collisions at $\sqrt{s_{N N}}=5.02$ and $8.16 \mathrm{TeV}$, publishing several results relative to forward photon [5, 6], $\pi^{0}$ [7-9] and neutron [10-12] production. However, in Run III, we will have the unique opportunity to directly measure forward production from high energy p-O and O-O collisions, in a configuration that is very similar to the interaction of an UHECR with an atmospheric nucleus, which is generally $\mathrm{N}$ or $\mathrm{O}$.

\section{The experiment}

The LHCf experiment consists of two small sampling calorimeters installed in two regions on the opposite sides of LHC Interaction Point 1 (IP1). These regions, called TArget Neutral absorber (TAN), are located at a distance of $141.05 \mathrm{~m}$ from IP1, after the dipole magnets that bend the two circulating beams. In this position, the LHCf experiment is capable of detecting the neutral particles that are produced in hadronic collisions with a pseudorapidity $\eta>8.4$. Each one of the two detectors, called Arm1 and Arm2, is made up by two square towers of $22 \mathrm{~W}$ and 16 GSO layers for a total length of $22 \mathrm{~cm}$, equivalent to $44 X_{0}$ and $1.6 \lambda_{I}$. Towers sizes are $20 \mathrm{~mm} \times 20 \mathrm{~mm}$ and $40 \mathrm{~mm} \times 40 \mathrm{~mm}$ for Arm1, $25 \mathrm{~mm} \times 25 \mathrm{~mm}$ and $32 \mathrm{~mm} \times 32 \mathrm{~mm}$ for Arm2. Energy resolution is better than $5 \%$ for $\gamma \mathrm{s}$ above $100 \mathrm{GeV}$ and about $40 \%$ for hadrons above $500 \mathrm{GeV}$. The transverse position of the incident particle is reconstructed using $4 \mathrm{X} / \mathrm{Y}$ imaging layers inserted at different depths. They are formed by $1 \mathrm{~mm}$ width GSO-bars in the case of Arm1 and by $160 \mu \mathrm{m}$ read-out pitch silicon microstrip detectors in the case of Arm2. Position resolution is better than $200 \mu \mathrm{m}$ for 


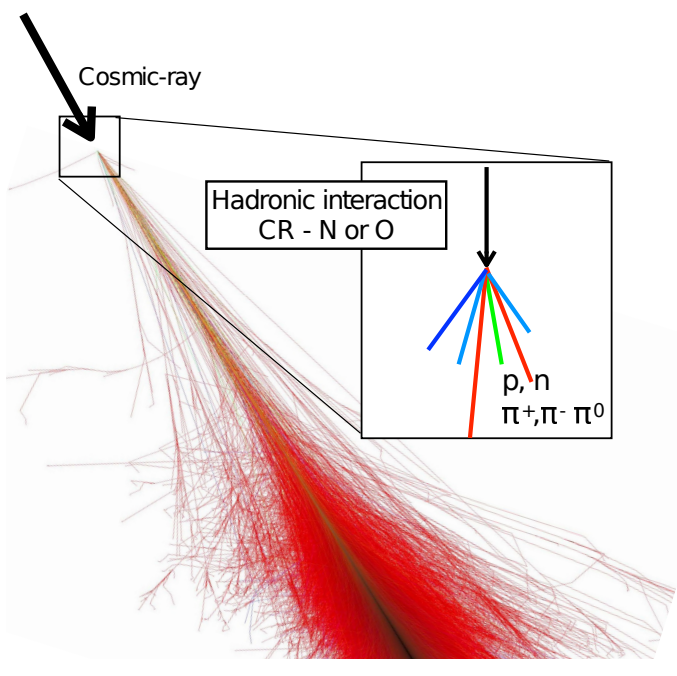

Figure 1: Schematic representation of an EAS generated by the interaction of an UHECR in the atmosphere.

$\gamma$ s above $100 \mathrm{GeV}$ and $1 \mathrm{~mm}$ for hadrons above $500 \mathrm{GeV}$. A more detailed description of detector performances is reported in [13]. In Run III the detectors will be exactly the same as in Run II, but there will be a few hardware upgrades. The major upgrade involves the Data AcQuisition (DAQ) system of the Arm2 silicon microstrip detector, which will allow for a ten times increase of the DAQ rate. In this way, it will be possible to acquire a ten times larger statistics in the same data-taking period, a fact that is very important for precise measurements of forward $\pi^{0}$ production.

\section{Scientific motivation for $\mathbf{p - O}$ collisions}

As schematically shown in Fig.1, the EAS formed by an UHECR in the atmosphere is originated by a first hadronic interaction of the cosmic ray with an atmospheric nucleus. Considering the chemical composition of the atmosphere, the target is generally a light nucleus, like $\mathrm{N}$ or O. Thus, hadronic interaction models used to simulate EASs can be accurately tuned by high energy calibration data where the target is a light nucleus. This configuration was not available in LHC Run I and II, where the only collisions useful for this application where p-p and p-Pb. The LHCf experiment had operations in these two configurations at different collisions energy and noticed, as expected, that forward production is strongly suppressed by the nuclear matter of the target in the case of $\mathrm{p}-\mathrm{Pb}$ respect to $\mathrm{p}-\mathrm{p}$ collisions. However, in order to estimate forward production in the realistic $\mathrm{p}-\mathrm{O}$ case, it is necessary to interpolate the measurements in these two configurations by using a proper theoretical model. All these problems will be overcame in Run III, where p-O collisions will be directly accessible at the LHC. To measure forward production in this configuration is one of the main scientific goal of the LHCf experiment. However, this will be the last chance that the experiment has to accomplish this goal, since the TAN region will be reshaped after Run III so that the detector should be completely redesigned to fit this new structure. Being this measurement so crucial for the cosmic-ray community, the LHCf collaboration sent a letter to the LHC committee, signed by about 100 researchers working in cosmic-ray and accelerator research field, to support LHCf operations in Run III with p-O collisions. 
Apart from the strong motivation related to cosmic-ray physics, there are additional reasons why forward production from $\mathrm{p}$-ion collisions can be more accurately studied in the case of $\mathrm{p}-\mathrm{O}$ rather than $\mathrm{p}-\mathrm{Pb}$ interactions. These are due to the fact that in $\mathrm{p}$-ion collisions forward production receives contributions from Ultra Peripheral Collision (UPC). UPC is an electromagnetic interaction, taking place for large impact parameters, between the incoming proton and a virtual photon of the target nucleus. As we can see from Fig.2 left, in the forward region UPC represents a serious background to ordinary $\mathrm{QCD}$ production in the case of $\mathrm{p}-\mathrm{Pb}$ collisions, but is negligible in case of $\mathrm{p}-\mathrm{O}$ collisions. This means that UPC in $\mathrm{p}-\mathrm{Pb}$ interactions is a contamination to our measurement, both in the general situation where we are interested in forward production to tune hadronic interaction models, and in the specific application where we want to later use these models to simulate EASs (since it is negligible in p-light ion interactions). When subtracting this background from our measurements, the large theoretical uncertainty on UPC (10\% from virtual photon flux and 10-50\% from protonphoton interaction) reflects on the final measurement. As shown in Fig.2 right, in the case of $\mathrm{p}-\mathrm{Pb}$ collisions, where the contributions from UPC and QCD are comparable, the final error band is dominated by the theoretical uncertainty on UPC, whereas in the case of $\mathrm{p}-\mathrm{O}$ collisions this source of uncertainty is negligible, since UPC is 1-2 order of magnitude smaller than QCD. This fact has
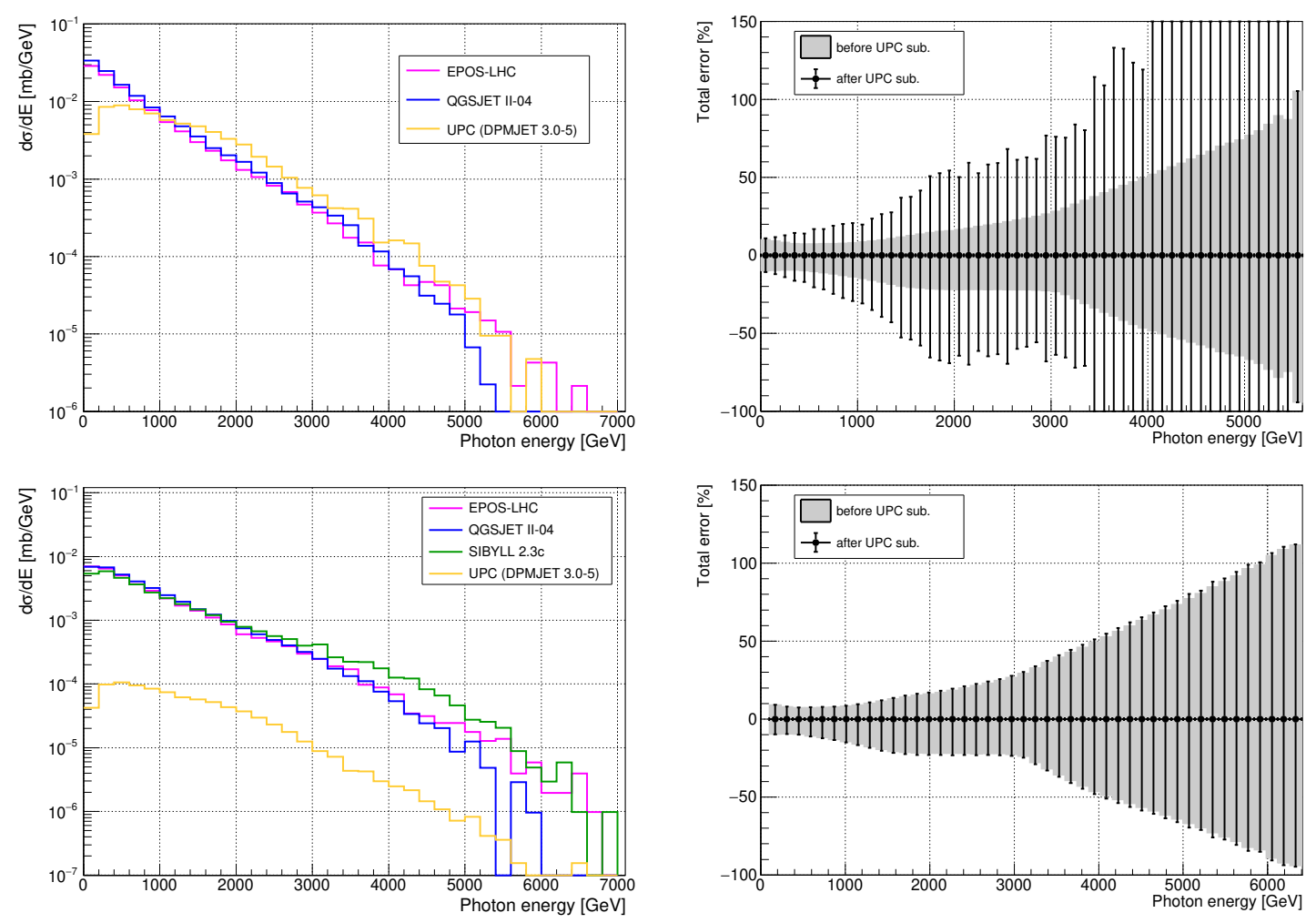

Figure 2: Impact of UPC contribution on forward photon production in $\eta>10.94$ (p-remnant side). Left: contribution from UPC together with the contribution from QCD, estimated using EPOS-LHC [14], QGSJET II-04 [15] and SIBYLL 2.3c [16] models. Right: impact of the UPC theoretical uncertainty on the final systematic uncertainty acting on forward photon spectra, after UPC background subtraction. Top figures refer to $\mathrm{p}-\mathrm{Pb}$ collisions at $\sqrt{s_{N N}}=8.16 \mathrm{TeV}$, bottom figures refer to $\mathrm{p}$-O collisions at $\sqrt{s_{N N}}=9.90 \mathrm{TeV}$. 

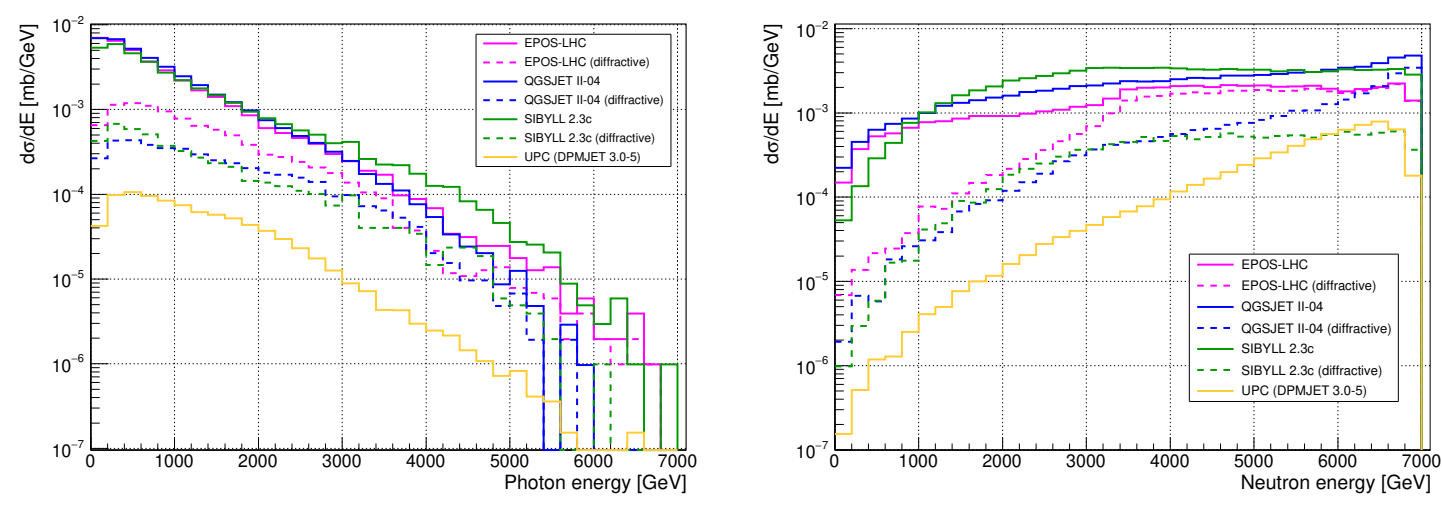

Figure 3: Impact of UPC contribution on forward production from p-O collisions at $\sqrt{s_{N N}}=9.90 \mathrm{TeV}$ (p-remnant side). The left figure represents photon in $\eta>10.94$, the right figure represents neutron in $\eta>10.76$. For each of the three models already shown in Fig.2, solid and dashed lines respectively represent inclusive production and diffractive production only.

important consequences for p-ion collisions, not only in terms of accurate measurements of QCD contributions to forward production, but also in terms of the ability to distinguish different QCD processes responsible for such a production, as described in the following.

In hadronic interaction models, diffractive and non-diffractive inelastic events are treated separately, so that an important indication for model tuning comes from separate measurements of forward production due to non-diffractive and different kinds of diffractive processes. As demonstrated by a preliminary result from Run II [17], the common data-taking and joint analysis of the LHCf ant ATLAS [18] experiments is a very powerful tool to accomplish this task in the case of p-p collisions. According to Monte Carlo studies [19], in a very simple approach diffractive events can be identified with a purity of almost $100 \%$ and an efficiency of about $50 \%$ by requiring a central veto in the ATLAS tracker, defined as zero charged tracks with $\mathrm{p}_{\mathrm{T}}>100 \mathrm{MeV}$ in $|\eta|<2.5$. However, due to the large UPC contribution that, having no activity in the central region, mimics a diffractive event, the LHCf-ATLAS joint analysis could not be successfully applied to the $\mathrm{p}-\mathrm{Pb}$ case. The situation is completely different in the $\mathrm{p}-\mathrm{O}$ case, since, as already observed, the UPC has a negligible contribution respect to QCD. As we can see from Fig.3, even considering only forward production due to diffractive processes, this is in any case much larger than the UPC background. Thus, thanks to the operations in Run III, it will be possible to separate the different mechanisms responsible for forward production also in the case of p-ion collisions.

\section{Prospects from Run III}

In June 2021, the LHC council approved p-O and O-O collisions in 2023/2024. The total time allocated for these operations is a about one week in total, but currently it is not defined how many days will be dedicated to $\mathrm{p}-\mathrm{O}$ and $\mathrm{O}-\mathrm{O}$ collisions and which will be the center-of-mass energy $(\sqrt{s}=$ $=5.52$ or $7 \times \mathrm{Z} \mathrm{TeV}$ ). For the $\mathrm{LHCf}$ collaboration, the ideal situation would be to have $\mathrm{p}-\mathrm{O}$ collisions at $\sqrt{s}=7 \times \mathrm{Z} \mathrm{TeV}$, and to have the chance to collect enough statistics both in the $\mathrm{p}-\mathrm{O}$ and $\mathrm{O}-\mathrm{O}$ configurations. The reason to have $\mathrm{p}-\mathrm{O}$ collisions at $\sqrt{s}=7 \times \mathrm{Z} \mathrm{TeV}\left(\sqrt{s_{N N}}=9.90 \mathrm{TeV}\right)$ is simply due to the fact that, operating at the highest energy available, the detector will have the maximum 

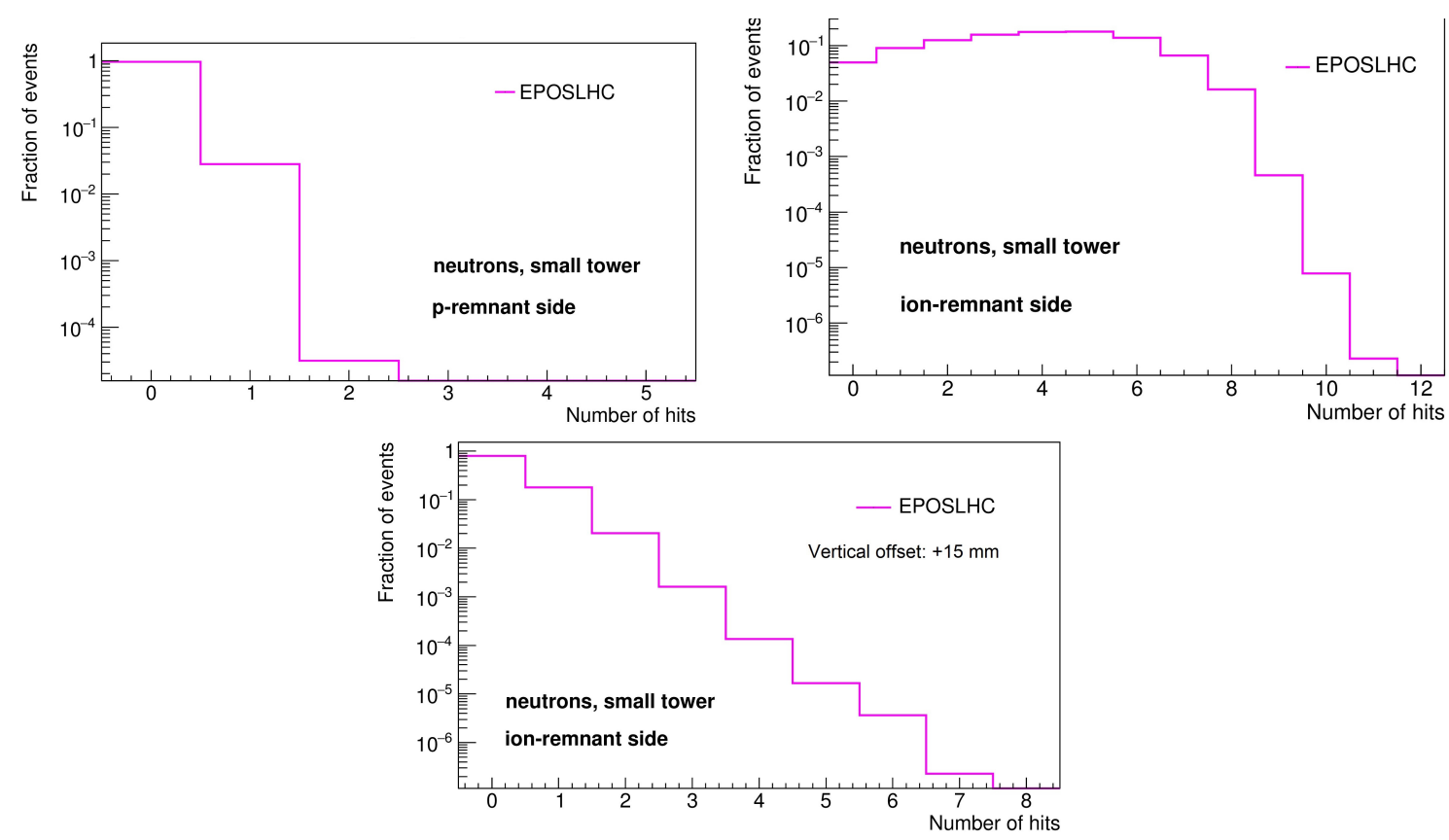

Figure 4: Neutron multiplicity in the small tower of the Arm2 detector in $\mathrm{p}-\mathrm{O}$ collisions at $\sqrt{s_{N N}}=9.90 \mathrm{TeV}$ estimated using the EPOS-LHC model. Top figures refer to the nominal detector position on the p-remnant (left) and O-remnant (right) sides. Bottom figure refers to the O-remnant side in the case that the detector is moved $15 \mathrm{~mm}$ higher respect to the nominal position.

coverage in the $\mathrm{p}_{\mathrm{T}}-\mathrm{x}_{\mathrm{F}}$ phase space. The reason to have both $\mathrm{p}-\mathrm{O}$ and $\mathrm{O}-\mathrm{O}$ collisions resides in the fact that it is important to study forward production in an equivalent UHECR-atmospheric nucleus interaction not only in the case of a primary proton, but also of a primary light nucleus. This consideration is supported by the fact that, using different hadronic interaction models, recent measurements of average composition qualitatively indicate that above $10^{18} \mathrm{eV}$ cosmic rays are composed by an admixture of light nuclei, but not by protons only.

In case of $\mathrm{p}-\mathrm{O}$ collisions at $\sqrt{s}=7 \times \mathrm{Z} \mathrm{TeV}$, the LHCf experiment is foreseen to acquire the required statistics, corresponding to an integrated luminosity of about $\mathrm{L}_{\mathrm{int}}=0.7 n b^{-1}$, in approximately two days of operations. The beam parameters are not yet fixed and a general agreement must be found between the requirements from different experiments. The ideal situation for the LHCf goals would be to operate with: a $145 \mu \mathrm{rad}$ downward crossing angle (to increase the pseudorapidity coverage), at least 24 colliding bunches (to increase the integrated luminosity in a fixed operation period), a 0.01-0.10 average number of collisions per bunch crossing (to avoid pile-up from the same bunch crossing), a minimum bunch spacing of $2 \mu s$ (to avoid pile-up from different bunch crossings), and a $\beta^{*}$ higher than $1 \mathrm{~m}$ (to strongly reduce the beam divergence).

The basic goal is to successfully operate in $\mathrm{p}-\mathrm{O}$ collisions on the p-remnant side, but in principle LHCf can also operate on the O-remnant side. However, as shown in Fig.4 top, simulation studies indicate that the average multiplicity in nominal position is much larger on the O-remnant side respect to the p-remnant side. Considering the good position resolution of the Arm2 detector, it is possible to reconstruct two particles simultaneously hitting the same calorimetric tower but it is very challenging to work with such a high multiplicity. Thus, in order to successfully operate on the 
O-remnant side, it is necessary to move the detector $15 \mathrm{~mm}$ respect to the nominal position, where, as we can see from Fig.4 bottom, multiplicity of secondaries strongly reduces. This configuration has obviously the disadvantage to limit the coverage of the forward region to a pseudorapidity $\eta<11$, but it still gives us a strong information on forward production on the O-remnant side without any additional hardware work. A similar solution can be adopted in O-O collisions.

\section{Conclusions}

The LHCf experiment successfully operated in LHC Run I and II with p-p and p-Pb collisions at different center-of-mass energies. This data allowed us to measure forward production of secondary particles, which is so important for the calibration of hadronic interaction models used to simulate EASs. However, these results are not exactly the equivalent to the first interaction of an UHECR with an atmospheric nucleus, which is generally a light nucleus. In Run III, the LHCf experiment will have the unique opportunity to measure forward production in such a configuration. In addition, thanks to $\mathrm{p}-\mathrm{O}$ and $\mathrm{O}-\mathrm{O}$ collisions, it will be possible to take data in the equivalent configuration of the first interaction of an UHECR with the atmosphere both in the case that the cosmic ray is a proton or an oxygen nucleus. This fact, together with the negligible impact of UPC background and with the powerful LHCf-ATLAS joint analysis, will lead to accurate forward measurements that will be crucial to tune hadronic interaction models used in cosmic-ray physics.

\section{References}

[1] P.A. Collaboration et al., The pierre auger cosmic ray observatory, Nuclear Instruments and Methods in Physics Research Section A: Accelerators, Spectrometers, Detectors and Associated Equipment 798 (2015) 172.

[2] M. Fukushima, Telescope array project for extremely high energy cosmic rays, Progress of Theoretical Physics Supplement 151 (2003) 206.

[3] L. Evans, The large hadron collider, New Journal of Physics 9 (2007) 335.

[4] O. Adriani, L. Bonechi, M. Bongi, G. Castellini, R. D’Alessandro, D. Faus et al., The LHCf detector at the CERN large hadron collider, Journal of Instrumentation 3 (2008) S08006.

[5] O. Adriani, L. Bonechi, M. Bongi, G. Castellini, R. D’Alessandro, A. Faus et al., Measurement of zero degree single photon energy spectra for proton-proton collisions at LHC, Physics Letters B 703 (2011) 128.

[6] O. Adriani, E. Berti, L. Bonechi, M. Bongi, R. d'Alessandro, M. Haguenauer et al., Measurement of forward photon production cross-section in proton-proton collisions at $\sqrt{s}$ $=13$ tev with the lhcf detector, Physics Letters B 780 (2018) 233.

[7] O. Adriani, L. Bonechi, M. Bongi, G. Castellini, R. D’Alessandro, K. Fukatsu et al., Measurement of forward neutral pion transverse momentum spectra for $\sqrt{s}=7 \mathrm{TeV}$ proton-proton collisions at the LHC, Physical Review D 86 (2012) 092001. 
[8] O. Adriani, E. Berti, L. Bonechi, M. Bongi, G. Castellini, R. D’Alessandro et al., Transverse-momentum distribution and nuclear modification factor for neutral pions in the forward-rapidity region in proton-lead collisions at $\sqrt{s_{N N}}=5.02 \mathrm{TeV}$, Physical Review C 89 (2014) 065209.

[9] O. Adriani, E. Berti, L. Bonechi, M. Bongi, R. D’Alessandro, M. Del Prete et al., Measurements of longitudinal and transverse momentum distributions for neutral pions in the forward-rapidity region with the LHCf detector, Physical Review D 94 (2016) 032007.

[10] O. Adriani, E. Berti, L. Bonechi, M. Bongi, G. Castellini, R. D’Alessandro et al., Measurement of very forward neutron energy spectra for $7 \mathrm{TeV}$ proton-proton collisions at the Large Hadron Collider, Physics Letters B 750 (2015) 360.

[11] O. Adriani, E. Berti, L. Bonechi, M. Bongi, R. D’Alessandro, S. Detti et al., Measurement of inclusive forward neutron production cross section in proton-proton collisions at $\sqrt{s}=13$ tev with the lhcf arm2 detector, Journal of High Energy Physics 2018 (2018) 1.

[12] O. Adriani, E. Berti, L. Bonechi, M. Bongi, R. D'Alessandro, S. Detti et al., Measurement of energy flow, cross section and average inelasticity of forward neutrons produced in $\sqrt{s}=13$ tev proton-proton collisions with the lhcf arm 2 detector, Journal of High Energy Physics 2020 (2020) 1.

[13] Y. Makino, A. Tiberio, O. Adriani, E. Berti, L. Bonechi, M. Bongi et al., Performance study for the photon measurements of the upgraded lhcf calorimeters with gd2sio5 (gso) scintillators, Journal of Instrumentation 12 (2017) P03023.

[14] T. Pierog, I. Karpenko, J. Katzy, E. Yatsenko and K. Werner, EPOS LHC: Test of collective hadronization with data measured at the CERN Large Hadron Collider, Physical Review C 92 (2015) 034906.

[15] S. Ostapchenko, Monte Carlo treatment of hadronic interactions in enhanced Pomeron scheme: QGSJET-II model, Physical Review D 83 (2011) 014018.

[16] R. Engel, F. Riehn, A. Fedynitch, T.K. Gaisser and T. Stanev, The hadronic interaction model sibyll-past, present and future, in EPJ Web of Conferences, vol. 145, p. 08001, EDP Sciences, 2017.

[17] T. LHCf and A. collaboration, Measurement of contributions of diffractive processes to forward photon spectra in pp collisions at $\sqrt{s}=13$ tev, ATLAS-CONF-2017-075 (2017) .

[18] G. Aad, E. Abat, J. Abdallah, A. Abdelalim, A. Abdesselam, O. Abdinov et al., The ATLAS experiment at the CERN large hadron collider, Journal of Instrumentation 3 (2008) .

[19] Q.-D. Zhou, Y. Itow, H. Menjo and T. Sako, Monte carlo study of particle production in diffractive proton-proton collisions at $\sqrt{s}=13$ tev with the very forward detector combined with central information, The European Physical Journal C 77 (2017) 1. 


\section{Full Authors List: LHCf Collaboration}

O. Adriani ${ }^{a, b}$, E. Berti $^{a, b}$, L. Bonechi $^{a}$, M. Bongi $^{a, b}$, R. D’Alessandro $^{a, b}$, G. Castellini $^{c}$, M. Haguenauer $^{d}$, $^{\prime}$

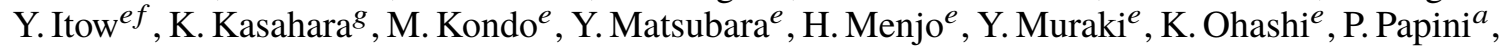

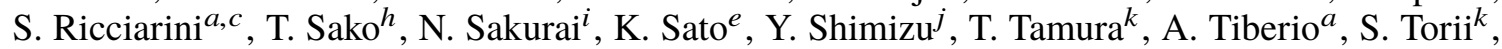
A. Tricomi ${ }^{l, m, n}$, W. C. Turner ${ }^{o}$, M. Ueno ${ }^{e}$, K. Yoshida ${ }^{g}$

${ }^{a}$ INFN Section of Florence - Florence, Italy

${ }^{b}$ University of Florence - Florence, Italy

${ }^{c}$ IFAC-CNR - Florence, Italy

${ }^{d}$ Ecole-Polytechnique - Palaiseau, France

${ }^{e}$ Institute for Space-Earth Environmental Research, Nagoya University - Nagoya, Japan

${ }^{f}$ Kobayashi-Maskawa Institute for the Origin of Particles and the Universe, Nagoya University - Nagoya, Japan

$g$ Faculty of System Engineering, Shibaura Institute of Technology - Saitama, Japan

${ }^{h}$ Institute for Cosmic Ray Research, University of Tokyo - Kashiwa, Japan

${ }^{i}$ Tokushima University - Tokushima, Japan

${ }^{j}$ Kanagawa University - Kanagawa, Japan

${ }^{k}$ RISE, Waseda University - Shinjuku, Tokyo, Japan

${ }^{l}$ INFN Section of Catania - Catania, Italy

${ }^{m}$ University of Catania - Catania, Italy

${ }^{n}$ CSFNSM - Catania, Italy

${ }^{o}$ LBNL - Berkeley, California, USA 